\title{
A self-assembling peptide hydrogel for ultrarapid 3D immunoassays
}

Alessandro Gori, Paola Gagni, Greta Bergamaschi, Alessandro Romanato, Marina Cretich

National Research Council of Italy, Italy

https://doi.org/10.17952/35EPS.2018.145

Microarray technology advent has set a new paradigm in the development of multiplexed assays for biomedical and many efforts were oriented to develop innovative surface chemistries able to balance a stable binding with retained functionality of immobilized bioprobes $[1,2,3]$. Hydrogels represent ideal systems bridging dry and wet conditions as biomolecules can be locally entrapped on analytical surfaces in their active form under solution mimetic conditions. However, some hydrogels characteristics still prevent their broad application for bioassays development. Particularly, a major issue is to match target diffusion rates through the gel matrix with stable probe entrapment since mass transport can be severely limited in dense cross-linked matrices.

In the present work, using micromolar concentrations of a self-assembling peptide we obtained a soft hydrogel matrix with controlled permeability properties for the straightforward confinement of biomolecular probes in arrayed 3D microenvironments. The self-assembling Q11 peptide (QQKFQFQFEQQ) is a fibrilforming amino acid stretch previously used as versatile nanostructured scaffold for several applications [4]. In our design we rationalized that working at low peptide concentrations was a necessary condition to obtain a low-viscosity hydrogel that could be handled for straightforward (micro)arrays production(Figure 1) [5]. Large biomolecules are entrapped within the fibrillary network due to their size whereas peptides can be immobilized by means of a co-assembly strategy with YF-Q11.

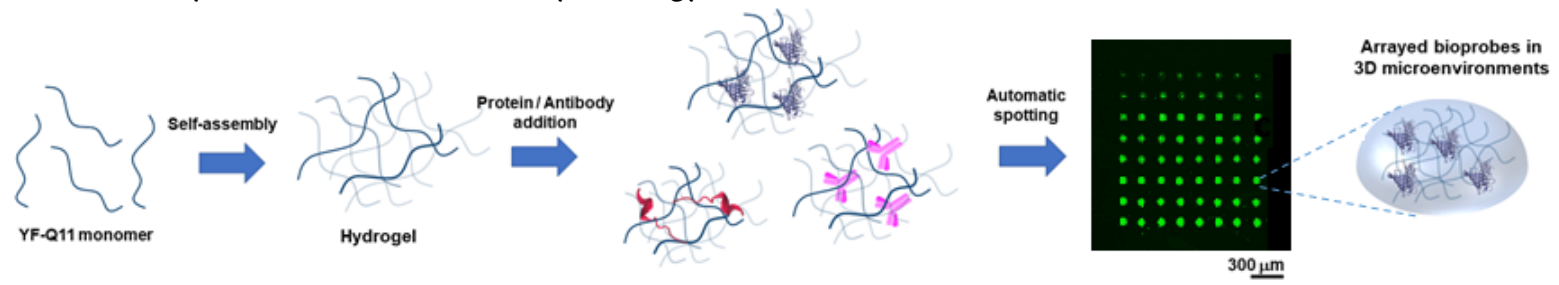

Figure 1: Overview of hydrogel bioassay strategy.

We used a slightly modified Q11 sequence (Ac-YFQQKFQFQFEQQ- $\mathrm{coNH}_{2}$, YF-Q11) that formed PMMA-adhesive soft hydrogels in a wide micromolar concentration range (25-500 $\mu \mathrm{M})$. Structural characterization of the new peptide by circular dichroism (CD) and ATR-FTIR analysis revealed that YF-Q11 preferentially shows a beta-sheet character, while spectrofluorimetric studies demonstrated that upon incubation at $40^{\circ} \mathrm{C}$ the spontaneous assembly proceeds rapidly and reaches a stable level within a convenient operative time frame (1-4 hours). Atomic force microscopy (AFM) confirm that peptide chains hierarchically aggregate in fibrillary structures that, in turn, self-organize into larger micrometer sized bundle of fibers (Figure 2 ). Rheology characterization of YF-Q11 hydrogel, showing that it behaves as a typical viscoelastic fluid under increasing strain excitation.

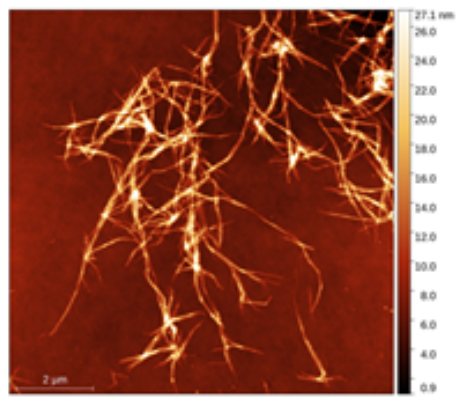

Figure 2: AFM representative image of 25 MM YF-Q11 showing the formation of entangled nanofibers.

To assess the feasibility of YF-Q11 hydrogel towards bioassay applications, we designed an experiment providing combined information on spotted gel permeation to biomolecules while monitoring its stability. To this aim we exploited a dual fluorescence detection: a Cy3-labelled YF-Q11 peptide $(0.1 \mu \mathrm{M})$ was co-assembled into 
different YF-Q11 solutions (25-500 $\mu \mathrm{M}$ range) to monitor the variation of fluorescence intensity of gel spots upon incubation of the slide in washing buffer at different times. Simultaneously, YF-Q11 spots containing Cy5-labelled biomolecules of representative molecular sizes (anti- $\alpha$-lactalbumin IgG, streptavidin and V5 peptide) were scanned for Cy5 residual fluorescence to monitor diffusion through the fibrillar network. The whole range of YF-Q11 hydrogels tested concentrations $(25-500 \mu \mathrm{M})$ showed a good stability over time. V5 peptide was fully permeable in all tested concentrations, while streptavidin freely diffuses below $100 \mu \mathrm{M}$; the same concentration allowed only for partial $\alpha$-lactalbumin antibody diffusion, which however was almost quantitative at $25 \mu \mathrm{M}$ (Figure 3).

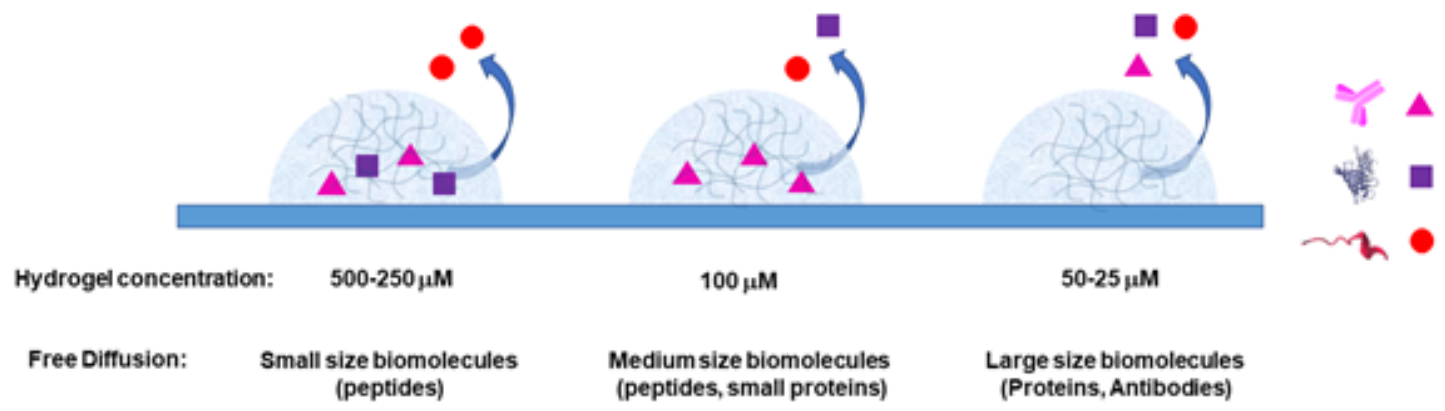

Figure 3: Graphical representation summary of hydrogel permeation to biomolecules.

Additionally, to verify that biomolecules are actually distributed in a 3D shell, confocal Raman imaging experiments were performed on an antibody-containing hydrogel with a concentration of $250 \mu \mathrm{M}$, results indicated that the labeled antibody was stably embedded in the hydrogel spots and uniformly distributed through the gel matrix (Figure 4). We then verified if these premises could translate into specific molecular recognition assays inside the hydrogel matrix.

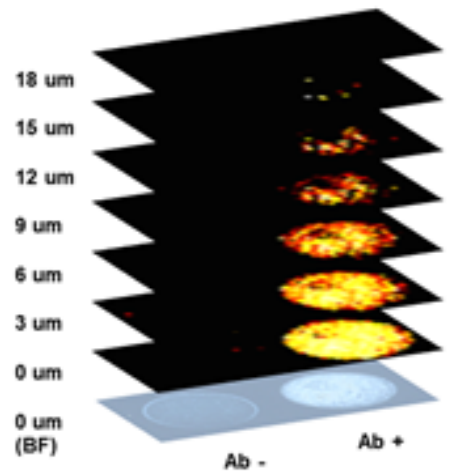

Figure 4: Confocal Raman imaging using a malachite-labelled antibody indicated it is stably embedded and uniformely distributed within the hydrogel spots.

To prove that, we designed symmetrical antibody-peptide recognition tests: in the first experiment we immobi-lized two different peptides (V5 and LAC) within the matrix and hydrogels were then probed with their specific Cy3/Cy5-antibodies. We also executed a specular assay in which we immobilized antibodies within the hydrogel. Both experiments showed that probe-specific recognition occurred only between respective antibody-peptide pairs (Figure 5). 
a)

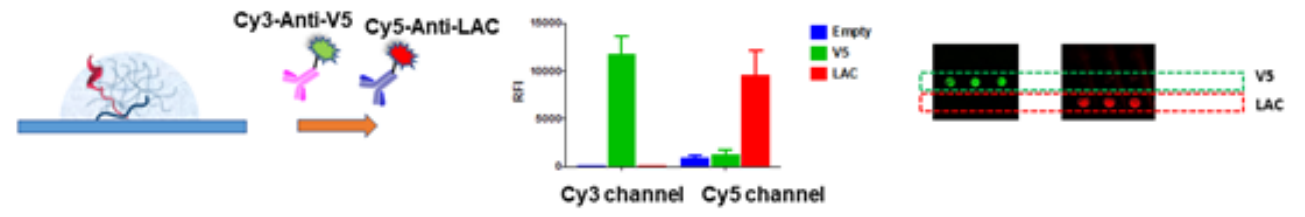

b)

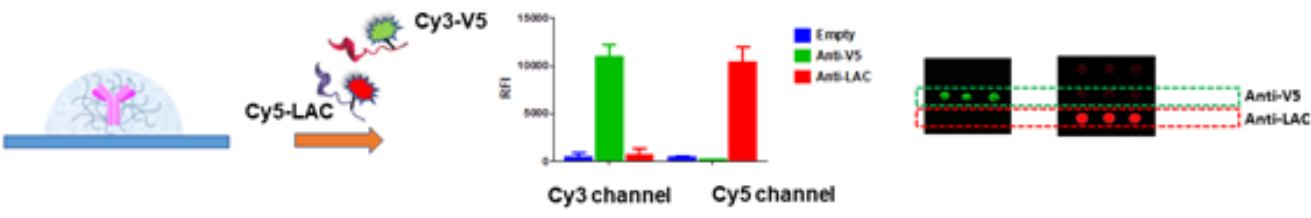

Figure 5: Fluorescence intensities detected in the Cy3 and Cy5 channels incubation of hydrogel spots with the respective probes.

As the final benchmark for our platform, we performed a real immunodiagnostic assay to detect Zika Virus (ZIKV) infection in human serum samples, confirming the actual feasibility of our system in the context of a real serological assay for infectious diseases. The total time required for this assay was $<10$ minutes, since the first recognition takes less than 5 minutes while incubation time for secondary antibody was kept at 2 minutes.

These results demonstrated that we obtained a stable and yet highly permeable nano-scaffold that enabled us to run fluorescence immunoassays under solution-like conditions in an ultrashort timespan. Remarkably, the supramolecular hydrogel forms rapidly and spontaneously, it is printable by a piezoelectric microarray spotter and it self-adheres on poly(methyl metacrylate) (PMMA) slides, so that neither post-spotting cross-linking nor polymerization is required for gel formation and surface attachment, greatly simplifying the fabrication process. Overall, our platform is user-friendly, robust and cost-effective and possesses a combination of favourable features which is unique in the area of 3D multiplexed bioassays in hydrogels.

\section{References}

1 A. Gori, M. Cretich, R. Vanna, L. Sola, P. Gagni, G. Bruni, M. Liprino, F. Gramatica, S. Burastero and M. Chiari, Anal. Chim. Acta, 2017, 983, 189-197.

2 A. Gori, L. Sola, P. Gagni, G. Bruni, M. Liprino, C. Peri, G. Colombo, M. Cretich and M. Chiari, Bioconjug. Chem., DOI:10.1021/acs.bioconjchem.6b00426.

3 L. Sola, P. Gagni, I. D’Annessa, R. Capelli, C. Bertino, A. Romanato, F. Damin, G. Bergamaschi, E. Marchisio, A. Cuzzocrea, M. Bombaci, R. Grifantini, M. Chiari, G. Colombo, A. Gori and M. Cretich, ACS Infect. Dis., 2018, 4, 998-1006.

4 J. S. Rudra, Y. F. Tian, J. P. Jung and J. H. Collier, Proc. Natl. Acad. Sci., 2010, 107, 622-627.

5 P. Gagni, A. Romanato, G. Bergamaschi, P. Bettotti, R. Vanna, C. Piotto, C. F. Morasso, M. Chiari, M. Cretich, A, Gori A Self-assembling Peptide Hydrogel for Ultrarapid 3D Bioassays, Nanoscale Adv., DOI:10.1039/C8NA00158H. 